\section{Exact BER of rectangular-constellation QAM subjected to asynchronous co-channel interference and Nakagami- $m$ fading}

\author{
X. Liu and L. Hanzo
}

The exact average bit error ratio (BER) of the rectangular quadrature amplitude modulation (QAM) constellation is studied in the context of asynchronous co-channel interference (CCI) and Nakagami- $m$ fading. A new formula is derived for the characteristic function of the $\mathrm{CCI}$, which requires no knowledge of the CCI distribution.

Introduction: Conventionally, the rectangular QAM (R-QAM) BER has been estimated by using various approximations or bounds. Most exact QAM BER results are obtained without taking into account the co-channel interference (CCI) [1,2]. When CCI is considered, exact BER analyses procedures are performed only for BPSK [3-5] and QPSK $[4,5]$ systems. Hence, the contribution of this Letter is that we derive an exact BER expression for R-QAM systems corrupted by both asynchronous CCI and Nakagami-m fading, while dispensing with the Gaussian distributed CCI assumption.

System model: We consider a general R-QAM system subjected to $K$ asynchronous co-channel interferers. The received signal $r(t)$ may be written as:

$$
\begin{aligned}
r(t)= & \sum_{k=0}^{K} h_{k}\left\{d_{k}^{I} b_{k}^{I}\left(t-\tau_{k}\right) \cos \left[\omega_{c}\left(t-\tau_{k}\right)+\theta_{k}+\phi_{k}\right]\right. \\
& \left.+d_{k}^{Q} b_{k}^{Q}\left(t-\tau_{k}\right) \sin \left[\omega_{c}\left(t-\tau_{k}\right)+\theta_{k}+\phi_{k}\right]\right\}+\eta(t)
\end{aligned}
$$

where $\omega_{c}$ and $\theta$ are the common carrier frequency and the carrier phase shift. As illustrated in [1], $2 d_{k}^{I}$ and $2 d_{k}^{Q}$ are the minimum distances between signal constellation points along the in-phase and quadraturephase axes, respectively. The in-phase and quadrature-phase data signals are given by $b_{k}^{I}(t)=\sum_{n=-\infty}^{\infty} b_{k, n}^{I} p T(t-n T)$ and $b^{Q}(t)=$ $\sum_{n=-\infty}^{\infty} b_{k, n}^{Q} p T(t-n T)$, respectively, where $b_{k, n}^{I}$ and $b_{k, n}^{Q}$ are in-phase and quadrature-phase data symbols, respectively. The symbol duration is denoted as $T$ and $p T(t)$ is the rectangular pulse having a duration of $T$. The $k$ th user's constellation size is $M_{k}=M_{k}^{I} \times M_{k}^{Q}$ and $\log _{2} M_{k}^{I}$ as well as $\log _{2} M_{k}^{Q}$ bits are Gray encoded and mapped onto the $k$ th user's inphase and quadrature-phase components [1], respectively. Hence, the in-phase and quadrature data symbols, $b_{k, n}^{I}$ and $b_{k, n}^{Q}$, are equiprobably selected from the set of $A_{k}^{I}=\left\{ \pm 1, \pm 3, \ldots, \pm\left(M_{k}^{I}-1\right)\right\}$ and $A_{k}^{Q}=$ $\left\{ \pm 1, \pm 3, \ldots, \pm\left(M_{k}^{Q}-1\right)\right\}$, respectively. The fading amplitude $h_{k}$ obeys the Nakagami- $m$ distribution having parameters $\left\{m_{k}, \Omega_{k}\right\}$, the fading phase $\phi_{k}$ and the time delay $\tau_{k}$ arch is uniformly distributed over $[0,2 \pi)$ and $[0, T)$, respectively. The additive white Gaussian noise (AWGN) is denoted by $\eta(t)$. We assume that the 0 th user is the desired one. In the case of coherent demodulation as well as perfect channel estimation, the in-phase and quadrature-phase decision statistics, $Z^{I}$ and $Z^{Q}$, are given by:

$$
\begin{aligned}
& Z^{I}=d_{0}^{I} h_{0} b_{0,0}^{I}+\sum_{k=1}^{K} h_{k}\left(X_{k}^{I} \cos \Delta_{k}+X_{k}^{Q} \sin \Delta_{k}\right)+\eta^{I} \\
& Z^{Q}=d_{0}^{Q} h_{0} b_{0,0}^{Q}+\sum_{k=1}^{K} h_{k}\left(X_{k}^{Q} \cos \Delta_{k}+X_{k}^{I} \sin \Delta_{k}\right)+\eta^{Q}
\end{aligned}
$$

where the phase shift difference $\Delta_{k}=-\omega_{c}\left(\tau_{k}-\tau_{0}\right)+\left(\theta_{k}-\theta_{0}\right)+\left(\phi_{k}-\right.$ $\left.\phi_{0}\right)$ is uniformly distributed over $[0,2 \pi)$. The noise components $\eta^{I}$ and $\eta^{Q}$ can be shown to be zero-mean Gaussian distributed random variables, while the random variables $X_{k}^{I}$ and $X_{k}^{Q}$ in (3) are defined as:

$$
\begin{aligned}
& X_{k}^{I}=d_{k}^{I}\left[b_{k,-1}^{I} v_{k}+b_{k, 0}^{I}\left(1-v_{k}\right)\right] \\
& X_{k}^{Q}=d_{k}^{Q}\left[b_{k,-1}^{Q} v_{k}+b_{k, 0}^{Q}\left(1-v_{k}\right)\right]
\end{aligned}
$$

where $v_{k}=\tau_{k} / T$ is the time delay normalised by the symbol duration.

BER analysis: Upon exploiting equation 57 of [6], we have the characteristic function (CF) of the in-phase CCI $I_{k}^{I}=h_{k}\left(X_{k}^{I} \cos \Delta_{k}+\right.$ $X_{k}^{Q} \sin \Delta_{k}$ ) conditioned on $X_{k}^{I}$ and $X_{k}^{Q}$ in the following form:

$$
\Phi_{I_{k}^{I} \mid X_{k}^{I}, X_{k}^{Q}}(\omega)={ }_{1} \mathbb{F}_{1}\left(m_{k} ; 1 ;-\frac{\Omega_{k}}{4 m_{k}}\left[\left(X_{k}^{I}\right)^{2}+\left(X_{k}^{Q}\right)^{2}\right] \omega^{2}\right)
$$

where ${ }_{1} \mathbb{F}_{1}(\alpha ; \beta ; x)$ is the confluent hypergeometric function [7]. Averaging $\Phi_{I_{l}^{I} \mid X_{k}^{I}, X_{l}^{Q}}(\omega)$ over the $k$ th interferer's data symbols and the time delay, we obtain the CF of $I_{k}^{I}, \Phi_{I_{k}}(\omega)$ as follows:

$$
\Phi_{I_{k}^{I}}(\omega)=\frac{1}{M_{k}^{2}} \sum_{b_{k,-1}^{I}, b_{k, 0}^{I} \in A_{k}^{I}} \sum_{b_{k,-1}^{Q}, b_{k, 0}^{Q} \in A_{k}^{Q}} \Phi_{I_{k}^{I} \mid \lambda_{0}, \lambda_{1}, \lambda_{2}}(\omega)
$$

where the coefficients $\lambda_{0}, \lambda_{1}$ and $\lambda_{2}$ are defined as:

$$
\begin{aligned}
& \lambda_{0}=\left(d_{k}^{I}\right)^{2}\left(b_{k, 0}^{I}\right)^{2}+\left(d_{k}^{Q}\right)^{2}\left(b_{k, 0}^{Q}\right)^{2} \\
& \lambda_{1}=\left(d_{k}^{I}\right)^{2} b_{k, 0}^{I}\left(b_{k,-1}^{I}-b_{k, 0}^{I}\right)+\left(d_{k}^{Q}\right)^{2} b_{k, 0}^{Q}\left(b_{k,-1}^{Q}-b_{k, 0}^{Q}\right) \\
& \lambda_{2}=\left(d_{k}^{I}\right)^{2}\left(b_{k,-1}^{I}-b_{k, 0}^{I}\right)^{2}+\left(d_{k}^{Q}\right)^{2}\left(b_{k,-1}^{Q}-b_{k, 0}^{Q}\right)^{2}
\end{aligned}
$$

The conditional CF, $\Phi_{I_{k}^{I} \mid \lambda_{0}, \lambda_{1}, \lambda_{2}}(\omega)$, may be shown to be given by:

$$
\begin{aligned}
& \Phi_{I_{k}^{I} \mid \lambda_{0}, \lambda_{1}, \lambda_{2}}(\omega) \\
& = \begin{cases}{ }_{1} \mathbb{F}_{1}\left(m_{k} ; 1 ;-\frac{\Omega_{k}}{4 m_{k}} \lambda_{0} \omega^{2}\right), & \text { if } \lambda_{2}=0 \\
\frac{x}{\lambda_{2}} \mathbb{F}_{1: 0 ; 1}^{1: 0 ; 1}\left(\begin{array}{ll}
{\left[\left(m_{k}\right): 1,1\right]:-;\left[\left(\frac{1}{2}\right): 1\right] ;} \\
{[(1): 1,1]:-;\left[\left(\frac{3}{2}\right): 1\right] ;}
\end{array}\right. & \\
\left.-\left(\lambda_{0}-\frac{\lambda_{1}^{2}}{\lambda_{2}}\right) \frac{\Omega_{k} \omega^{2}}{4 m_{k}},-\frac{\Omega_{k} \omega^{2} x^{2}}{4 \lambda_{2} m_{k}}\right)\left.\right|_{\lambda_{1}} ^{\lambda_{1}+\lambda_{2}} & \text { if } \lambda_{2} \neq 0\end{cases}
\end{aligned}
$$

where

$$
\begin{aligned}
& \mathbb{F}_{C: D^{(1)} ; \ldots ; D^{(n)}}^{A: B^{(1)} ; \ldots B^{(n)}} \\
& \left.\qquad \begin{array}{l}
{\left[(a): \theta^{(1)}, \ldots, \theta^{(n)}\right]:\left[\left(b^{(1)}\right): \phi^{(1)}\right] ; \ldots ;\left[\left(b^{(n)}\right): \phi^{(n)}\right] ;} \\
{\left[(c): \psi^{(1)}, \ldots, \psi^{(n)}\right]:\left[\left(d^{(1)}\right): \delta^{(1)}\right] ; \ldots ;\left[\left(d^{(n)}\right): \delta^{(n)}\right] ;}
\end{array} x_{1}, \ldots, x_{n}\right)
\end{aligned}
$$

is the generalised Lauricella function of $n$ variables [7] and $\left.f(x)\right|_{x_{1}} ^{x_{2}}=$ $f\left(x_{2}\right)-f\left(x_{1}\right)$. On defining the total in-phase interference plus noise term as $\xi^{I}=\sum_{k=1}^{K} I_{k}^{I}+\eta^{I}$, its cumulative distribution function (CDF) $F_{\xi}(x)$ can be shown to be:

$$
F_{\xi^{I}}(x)=\frac{1}{2}+\frac{1}{\pi} \int_{0}^{\infty} \frac{\sin (\omega x)}{\omega} \Phi_{\xi^{I}}(\omega) d \omega
$$

Extending the AWGN result of [1] to the scenarios in the presence of $\mathrm{CCI}$ and noise, the conditional error probability $P_{b \mid h 0}^{I}(u)$ of the $u$ th bit of the in-phase component can be expressed as follows:

$$
\begin{aligned}
P_{b \mid h_{0}}^{I}(u)= & \frac{1}{M_{k}^{I}} \sum_{l=0}^{\left(1-2^{-u}\right) M_{k}^{I}-1} \\
& \times\left\{(-1)^{\left\lfloor l 2^{u-1} / M_{k}^{I}\right\rfloor}\left(2^{u-1}-\left\lfloor\frac{l 2^{u-1}}{M_{k}^{I}}+\frac{1}{2}\right\rfloor\right)\right. \\
& \left.\times\left[2\left(1-\mathrm{F}_{\xi^{I}}\left((2 l+1) d_{0}^{I} h_{0}\right)\right)\right]\right\}
\end{aligned}
$$

On averaging $P_{b \mid h_{0}}^{I}(u)$ over $h_{0}$ and applying Parseval's theorem [8], we obtain the error probability $P_{b}^{I}(u)$ of the $u$ th bit of the in-phase component in the form of:

$$
\begin{aligned}
P_{b}^{I}(u)= & \frac{1}{2}-\frac{2}{\pi M_{k}^{I}} \int_{0}^{\infty} \frac{\Phi_{\psi^{I}}(\omega)}{\omega} \sum_{i=0}^{\left(1-2^{-u}\right) M_{k}^{I}-1} \\
& \times\left\{(-1)^{\left\lfloor 2^{u-1} / M_{k}^{I}\right\rfloor}\left(2^{u-1}-\left\lfloor\frac{l 2^{u-1}}{M_{k}^{I}}+\frac{1}{2}\right\rfloor\right)\right. \\
& \left.\times \Im\left\{\Phi_{h_{0}}\left[(2 l+1) d_{0}^{I} \omega\right]\right\}\right\} d \omega
\end{aligned}
$$

where $\Im\left\{\Phi_{h_{0}}(\omega)\right\}$ is the imaginary part of the CF of the desired user's fading amplitude, $h_{0}$, which is given by Table II of [8]. Following the same approach, we may derive the error probability $P_{\tilde{b}}^{Q}(u)$ of the $u$ th bit of the quadrature-phase component in the same form by replacing all symbols having the superscript $I$ by their counterparts with the superscript $Q$ in (14)

Finally, the average BER of $M$-ary general R-QAM can be obtained by averaging the error probabilities over the in-phase and quadraturephase components [1]. When there is no interference, i.e. we have $K=0$, and (14) reduces to the single-user results of [2]. As expected, 
when only BPSK or QPSK are considered, (14) reduces to the results of [5].

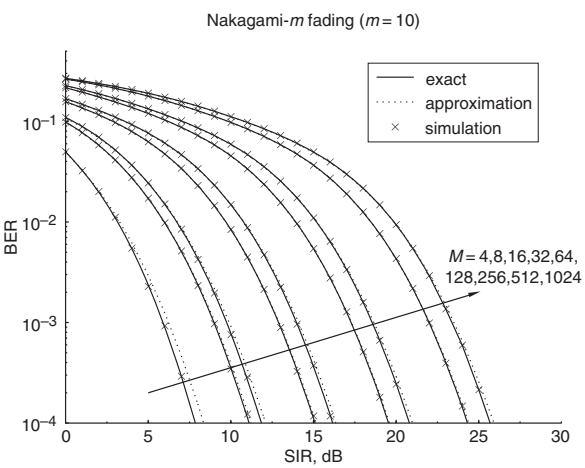

Fig. 1 BER against per-bit SIR in R-QAM system subjected to asynchronous CCI and Nakagami-m fading

Average power of each interferer is common and they experience the same fading distribution as the desired signal, i.e. $m_{k}=10$. All users have same constellation size, i.e. $M_{k}=M$. Minimum distances between signal points of in-phase and quadrature-phase components are the same, i.e. $d_{k}^{T}=d_{k}^{O}$. Number of interferers $K=6$. Background noise ignored

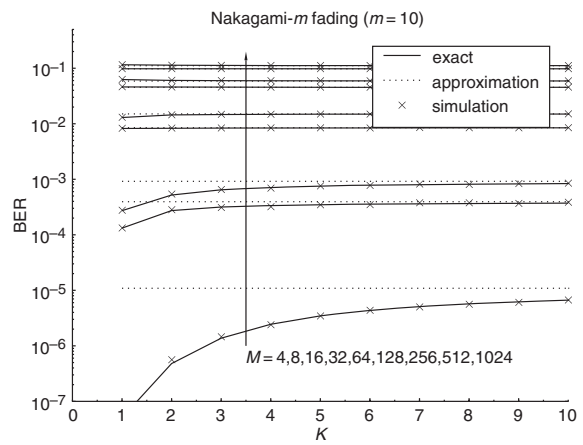

Fig. 2 BER against number of interferers $K$ in R-QAM system subjected to asynchronous $C C I$ and Nakagami-m fading

Average power of each interferer is common and they experience the same fading distribution as the desired signal, i.e. $m_{k}=10$. All users have same constellation size, i.e. $M_{k}=M$. Minimum distances between signal points of in-phase and quadrature-phase components are the same, i.e. $d_{k}^{I}=d_{k}^{Q}$. Per-bit SIR, $10 \mathrm{~dB}$. Background noise ignored

Results: We define the per-bit signal-to-interference ratio (SIR) as:

$$
\mathrm{SIR}=\frac{1}{\log _{2} M_{0}} \frac{\left[\left(d_{0}^{I}\right)^{2}+\left(d_{0}^{Q}\right)^{2}\right] \Omega_{0}}{\sum_{k=1}^{K}\left[\left(d_{k}^{I}\right)^{2}+\left(d_{k}^{Q}\right)^{2}\right] \Omega_{k}}
$$

Since the evaluation of the effects of CCI on the QAM BER is the main objective of our analysis, we assume that the effects of noise are negligible. Fig. 1 shows average BER performance against per-bit SIR expressed in dB. Fig. 2 shows average BER performance against the number of interferers. As seen in both Figures, the results calculated by our exact BER analysis and the simulation results match for various constellation sizes. On the other hand, the GA overestimates the average BER, especially when the constellation size is small and the per-bit SIR value is high or the number of interferers is small.

Acknowledgements: The financial support of the EPSRC, UK, and of the EU under the auspices of the Phoenix and Newcom projects, is gratefully acknowledged.

(C) The Institution of Engineering and Technology 2006

11 April 2006

Electronics Letters online no: 20060974

doi: 10.1049/el:20060974

X. Liu and L. Hanzo (School of Electronics and Computer Science, University of Southampton, SO17 1BJ, United Kingdom)

E-mail:1h@ecs.soton.ac.uk

\section{References}

1 Cho, K., and Yoon, D.: 'On the general BER expression of one- and twodimension amplitude modulations', IEEE Trans. Commun., 2002, 50, pp. 1074-1080

2 Yoon, D., and Cho, K.: 'General bit error probability of rectangular quadrature amplitude modulation', Electron. Lett., 2002, 38, pp. 131-133

3 Hamdi, K.A.: 'Exact probability of error of BPSK communication links subjected to asynchronous interference in Rayleigh fading environment', IEEE Trans. Commun., 2002, 50, pp. 1577-1579

4 Giorgetti, A., and Chiani, M.: 'Influence of fading on the Gaussian approximation for BPSK and QPSK with asynchronous cochannel interference', IEEE Trans. Wirel. Commun., 2005, 4, pp. 384-389

5 Sivanesan, K., and Beaulieu, N.C.: 'Exact BER analyses of Nakagami/Nakagami CCI BPSK and Nakagami/Rayleigh CCI QPSK systems in slow fading', IEEE Commun. Lett., 2004, 8, pp. 45-47

6 Shi, Q., and Latva-Aho, M.: 'Accurate bit-error rate evaluation for synchronous MC-CDMA over Nakagami- $m$-fading channels using moment generating functions', IEEE Trans. Wirel. Commun., 2005, 4, pp. $422-433$

7 Srivastava, H.M., and Karlsson, P.W.: 'Multiple Gaussian hypergeometric series' (Ellis Horwood, 1985)

8 Annamalai, A., Tellambura, C., and Bhargava, V.K.: 'Equal-gain diversity receiver performance in wireless channels', IEEE Trans. Commun., 2000, 48, pp. $1732-1745$ 